\title{
Research on Image Fusion for Visual and PMMW Images Using NSCT and Region Detection
}

\author{
Shi Tang ${ }^{1}$, Jintao Xiong, Jianyu Yang and Xinliang Peng
}

\begin{abstract}
In this paper, a novel image fusion algorithm based on Nonsubsampled Contourlet Transform (NSCT) and image segmentation is proposed, for the application of Passive Millimeter Wave (PMMW) concealed objects detection. PMMW and visual images are fused to get a better presentation of original images, maintaining both visual background and concealed object detected in PMMW images. This algorithm combines the extraction of Region of Interest (ROI) and the NSCT based fusion. The experimental results show that the proposed algorithm has better fusion performance compared with traditional methods.
\end{abstract}

Keywords: image fusion • Passive Millimeter Wave (PMMW) image • Nonsubsampled Contourlet Transform (NSCT) • image segmentation

\section{Introduction}

The difference of millimeter energy radiated by objects is detected by Passive Millimeter Wave (PMMW) imaging system, to provide PMMW images in which the metal targets can be distinguished from the background. Metal targets even covered by objects like clothes and leaves, can be detected. These features allow the PMMW imaging system to be widely used in concealed object detection $[1,2]$. However, compared with visual images, the visual quality of PMMW images is limited by the low spatial resolution and the severe blurring effect. Therefore, the fusion of visual and PMMW images, integrates the complementary information and reduces the redundancy, so that the concealed objects can be presented in the visual background with better visual quality [3].

\footnotetext{
${ }^{1}$ Shi Tang $(\square)$

School of Electronic Engineering, University of Electronic Science and Technology of China, Chengdu, China

e-mail: tangshi0429@163.com
} 
In recent years, Region Based Method and Multi-scale Decomposition Based Method are used by some scientists to fuse multi-source images. A combination of wavelet transform, edge based image segmentation and regional edge feature is used to generate a fusion map in literature $[5,6]$. These methods extremely depend on the segmentation accuracy, which is determined by a hard chosen gray value threshold. In addition, regional edge features are not very reliable when used as fusion guidance. Some other segmentation technics, such as fuzzy k-means clustering and frame difference, together with multi-scale decomposition are used to fuse visual and IR images [7, 8]. Although segmentation results do help the fusion, the visual background is still interfered by unwanted information from IR images. The main problems of these existing fusion algorithms can be summarized as:

- Image segmentation is unable to extract the true target areas.

- Quality of fusion is limited by the lack of protection of visual background.

The algorithm proposed can be used to solve problems mentioned above by taking the following steps: firstly using Mean Shift Segmentation [9] to extract the regions of interest in PMMW images, then adopting NSCT [10] based fusion to generate a fused ROI, finally embedding the fused ROI back into the visual images at the corresponding positions. The proposed algorithm has these advantages:

- Extracting the ROI in PMMW images accurately.

- Using NSCT to provide better details and direction descriptions of images.

- Only fusing the ROI so that more visual background is remained.

\section{Basic Theories}

\subsection{Extraction of Region of Interest}

The extraction of region of interest is applied using image segmention and the prior knowledge that the brightest area in PMMW image corresponds to metal object (the target). Mean Shift Segmentation is based on the gray value of image, and it segments images by conducting Mean Shift process to every pixel rather than set a threshold according to the gray value histogram. According to literature [9], the Mean Shift vector is

$$
M_{h}(x)=\frac{\sum_{i=1}^{n} G\left(\frac{x_{i}-x}{h}\right) w\left(x_{i}\right) x_{i}}{\sum_{i=1}^{n} G\left(\frac{x_{i}-x}{h}\right) w\left(x_{i}\right)}-x
$$


where $G(x)$ is a kernel which could be normal or uniform, and $h$ controls the size of range window, and $w\left(x_{i}\right) \geq 0$ is the weight assigned to the sample point $x_{i}$ which corresponds to the spatial window. Denote the first term on the right of equation (1) as $m_{h}(x)$. When given an initial point $x$, a kernel $G(X)$ and tolerable error $\varepsilon$, Mean Shift procedure is done by carrying the iteration below

1. Compute $m_{h}(x)$ according to (1);

2. Assign the value of $m_{h}(x)$ to $x$;

3. If $\left\|m_{h}(x)-x\right\|<\varepsilon$, end the iteration; else, return to step 1.

The basic idea of Mean Shift image segmentation is conducting Mean Shift procedure to each pixel of image, and classing pixels with the same convergence value and neighboring position together. The detail steps of segment is followed

1. Conduct Mean Shift iteration to each pixel $x_{i, j}$ of the image;

2. Assign $z_{i, j}=\left(x_{i, j}, y_{i, j}\right)$, where $y_{i, j}$ is the convergence value of $x_{i, j}$;

3. Set all $z_{i, j}$ with same $y_{i, j}$ value and neighboring position $x_{i, j}$ the same class L;

4. Compute convergence mean value of each class as $m_{L}=\sum_{y_{i, j} \in L} y_{i, j} / N$.

Use equation (2) to decide if $y_{i, j}$ value is the same

$$
\left|y_{i, j}-y_{i+s, k+t}\right| \leq K
$$

where $\mathrm{K}$ is an empirical constant between 0.01 to 0.04 after image pixel value is normalized to $[0,1]$.When (2) is satisfied, we consider $y_{i, j}$ have the same value.

After segmentation, the PMMW image is divided into several regions with different $m_{L}$. Normally, the target is often brighter (with larger $m_{L}$ ) and not distributed near the boundaries of the PMMW image. Using this prior knowledge, nontarget areas can be deleted .The regional image can be turned into a binary image in which pixel value of target is ' 1 ' while the rest is ' 0 '. A rectangular area from the binary image that just covers all the ' 1 's is designated and denoted as Map . A rectangular area with the same position and size as the Map , is obtained from the PMMW image as the ROI. The flowchart of extraction of ROI is show in Fig. 1.

\subsection{Nosubsampled Contourlet Transform}

NSCT uses nonsubsampled pyramid (NSP) and nonsubsampled directional filter bank (NSDFB) to achieve multi-scale decomposition with better frequency selec- 
tivity and regularity [10]. The NSCT is shift-invariant and this quality ensures that the bandpass and lowpass subbands have the same size. Fig.2 (a) shows the NSP and NSDFB structure that implements the NSCT, and Fig.2 (b) shows the idealized frequency partitioning obtained with the proposed structure.

The bandpass and lowpass subbands images correspond to fine scale and coarse scale, respectively. Thus, the point of fusion based on NSCT is to use different fusion rules in different subbands. The advantage of this is that we can chose to keep detail or approximation information in each subbands. The specific fusion strategy is described in section 3 .

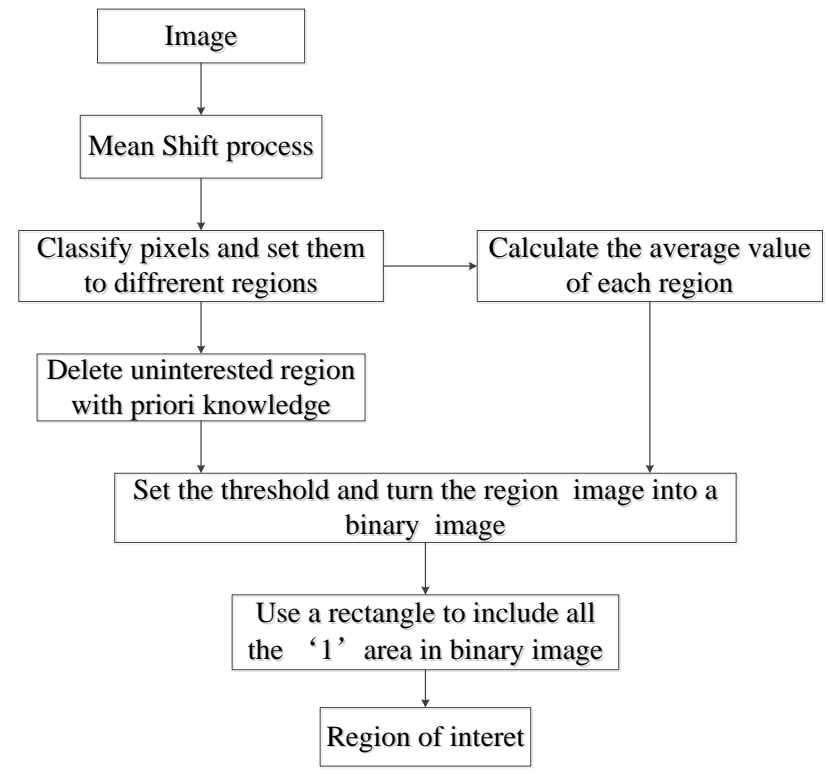

Fig. 1 The flow chart of extraction of ROI using Mean Shift Segmentation

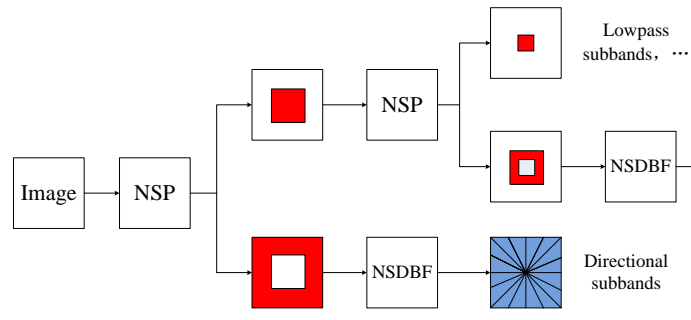

(a)

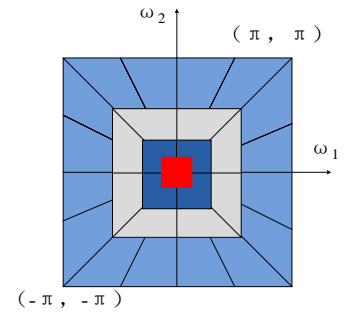

(b)

Fig. 2 The diagram of Nonsubsampled Contourlet Transfom, (a) the NSP and NSDFB structure (b) the idealized frequency partitioning 


\subsection{PMMW Image Enhancement and the ROI Embedding}

In image fusion, the fact that metal objects are always bright in PMMW image makes them hard to be noticed when the corresponding area of visual image is also very bright. Image enhancement of PMMW is able to help this special situation, and the enhancement is carried using equation (3)

$$
D_{B_{-} \text {new }}=k \cdot D_{B} \cdot \operatorname{Map} / \max \left(D_{B}\right)
$$

where $D_{B}$ is the lowpass subband obtained from NSCT decomposing the ROI area of PMMW image, Map is mentioned in 2.1, and $k$, which is chosen between 1.2 to 1.6 , is a constant to adjust the strength of enhancement.

When embedding the fused ROI back into the visual image, there always are salient boundary effects around the ROI area. This boundary effect can be eliminated by using the image blending algorithm in [11]. First mark the overlapping area between fused ROI and visual image, as shown in Fig.3 (a). Then calculate the new overlapping area by weighting method as equation (4)

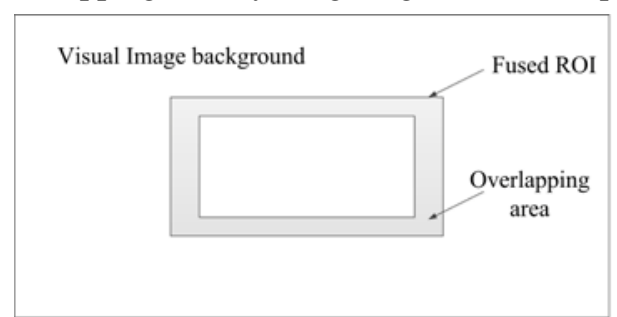

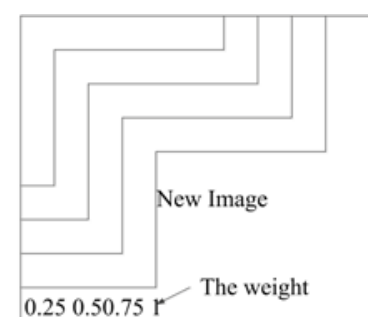

(b)

Fig. 3 The diagram of eliminating boundary effect. (a) the overlapping area, (b) the weight

$$
N(x, y)=\alpha \cdot I(x, y)+(1-\alpha) \cdot C(x, y)
$$

where $\mathrm{N}$ denotes the new overlapping area and $\alpha$ denotes the weight. I and $\mathrm{C}$ in equation (4) denote the overlapping area from fused ROI and from visual image, respectively. Empirically, the weight $\alpha$ is chosen as

$$
\boldsymbol{\alpha}=[0.25,0.5,0.75,1]^{T}
$$

\section{Process of Fusion Algorithm}

Different fusion rules are applied in bandpass and lowpass subbands images of the ROI. Weighted average merging method [12] is used to fuse the bandpass subbands to keep more detail and directional information. The WAMM is 


$$
D_{F}(p)= \begin{cases}w_{\max } D_{A}(p)+w_{\min } D_{B}(p) & E_{A}(p) \geq E_{B}(p) \\ w_{\min } D_{A}(p)+w_{\max } D_{B}(p) & E_{A}(p)<E_{B}(p)\end{cases}
$$

where $D_{A}(p)$ and $D_{B}(p)$ are the bandpass subbands images of ROI and the corresponding area of visual image, respectively. The $w_{\min }$ and $w_{\max }$ is calculated as equation (8). $E_{A}(p)$ and $E_{B}(p)$ represent the region energy

$$
E(p)=\sum_{s \in S, t \in T} w(s, t)[D(m+s, n+t, j, l)]^{2}
$$

where the weight $w$ is a Gaussian window.

$$
\left\{\begin{array}{cc}
w_{\min }=0, w_{\max }=1 & M_{A B}(p)<T \\
w_{\min }=\frac{1}{2}-\frac{1}{2}\left(\frac{1-M_{A B}}{1-T}\right), w_{\max }=1-w_{\min } & \text { other }
\end{array}\right.
$$

The $M_{A B}(p)$ in equation (8) is noted as match measurement

$$
M_{A B}(p)=\frac{2 \sum_{s \in S, t \in T} w(s, t) D_{A}(m+s, n+t, j, l) D_{B}(m+s, n+t, j, l)}{E_{A}(p)+E_{B}(p)}
$$

In the fusion of lowpass subbands, it is vital to choose coefficients have large region energy because larger region energy give more weight to the approximation of source image.

$$
D_{F}(p)= \begin{cases}D_{A}(p) & E_{A}(p)>E_{B}(p) \\ D_{B}(p) & E_{A}(p)<E_{B}(p) \\ \left(D_{A}(p)+D_{B}(p)\right) / 2 & E_{A}(p)=E_{B}(p)\end{cases}
$$

The whole fusion algorithm procedure is as followed:

1. Extract ROI from the PMMW image and enhance the ROI;

2. Get the subbands images using NSCT on the ROI and corresponding area in visual image;

3. Fuse the subbands images using different rules, then perform inverse NSCT to obtain the fused ROI;

4. Embed the fused ROI into the visual image.

\section{Results and Discussions}

In concealed object detection, the reveal of target feature in the PMMW image is as important as fusion quality. We combine objective and subjective evaluation to 
access the fusion result. The subjective evaluation is mainly based on the perception by human visual system of observers. The objective evaluation indices include entropy, average gradient, mutual information, spatial frequency and edge based similarity measure [13, 14, 15].

Two sets of registered PMMW and visual images are used to test the performance of the proposed algorithm, as shown in Fig.5 (a) and (b) and Fig.6 (a) and (b). The PMMW images we used are different at imaging condition and quality, so that the reliability of fusion is tested. The level of NSCT decomposition is 3 and all the experiments are carried out using MATLAB 7.11.0 on PC.

\begin{tabular}{|c|c|c|}
\hline (a)visual image & (b)PMMW image & (c)segmentation result \\
\hline (d)ROI & (e)wavelet fusion & (f)Contourlet fusion \\
\hline (g)NSCT fusion & ee proposed metho & \\
\hline
\end{tabular}

Fig. 4 Fusion results of PMMW and visual images with a hidden car as the target

$\begin{array}{llll}\text { (a)visual image } & \text { (b)PMMW image } & \text { (c)segmentation result } & \text { (d)delete background } \\ \text { (e)wavelet fusion } & \text { (f)Contourlet fusion } & \text { (g)NSCT fusion } & \text { (h)the proposed method }\end{array}$

Fig. 5 Fusion results of the bad imaged PMMW image and visual image with a hidden knife

To compare with, traditional fusion including wavelet, contourlet and NSCT based methods [16] are used. All the experiment results are shown in Fig.5 and Fig.6 whereas the evaluation indices are shown in Table 1. From Fig.5 (e) to Fig.5 (h), it is clearly that the proposed algorithm has the best fusion quality. The hidden car can be seen in all fusion results but Fig.5 (h) excels. The results from wavelet and Contourlet contain too much redundancy in the non-target area. The NSCT based fusion has a better result but the clarity and contrast is limited. The proposed method also has the best IE, MI, AG and EA as shown in Table 1 result 1. Especially the MI overwhelmingly excels because the fusion result remains a large part of visual background. 
Different situation occurs in the fusion of Fig.6 (a) and (b), since this PMMW image is poorly imaged. Beside of the knife, the background as well as a part of human body, is also extremely bright in this PMMW image. Wavelet, Contourlet and NSCT based method fuse images without deleting false target area in PMMW image. Therefore, Fig.6 (e) to (g) are unacceptable despite of the good objective evaluation indices shown in Table 1 . result 2 . With extraction of ROI before fusion, the proposed method successfully captures the hidden knife and fuses it into the visual background.

Table 1.Objective evaluation results

\begin{tabular}{|l|c|c|c|c|c|c|c|c|}
\hline \multirow{2}{*}{ Method } & \multicolumn{4}{|c|}{ Objective Evaluation Result 1 } & \multicolumn{4}{c|}{ Objective Evaluation Result 2} \\
\cline { 2 - 9 } & $\boldsymbol{I E}$ & $\boldsymbol{M I}$ & $\boldsymbol{A G}$ & $\boldsymbol{E A}$ & $\boldsymbol{I E}$ & $\boldsymbol{M I}$ & $\boldsymbol{A G}$ & $\boldsymbol{E A}$ \\
\hline Wavelet & 6.665 & 2.326 & 6.197 & 0.419 & 7.453 & 4.016 & 6.798 & 0.390 \\
\hline Contourlet & 7.105 & 2.267 & 8.251 & 0.564 & 7.799 & 3.689 & 9.634 & 0.398 \\
\hline NSCT & 7.282 & 3.735 & 9.146 & 0.617 & 7.151 & 3.556 & 9.230 & 0.500 \\
\hline Proposed & 7.603 & 6.445 & 9.287 & 0.619 & 7.271 & 5.151 & 5.870 & 0.476 \\
\hline
\end{tabular}

\section{Conclusions}

In this paper, a novel image fusion algorithm is proposed for PMMW concealed object detection in which visual and PMMW images are fused to synthesis complementary information. The significant improvement of this proposed algorithm comes from the combination of interesting area extraction and NSCT based fusion. Therefore, it has some feature level fusion characteristics while still providing good pixel level fusion quality. Experiments are performed to test the proposed fusion algorithm, and both the objective and subjective evaluation results demonstrate that this novel fusion algorithm can achieve better fusion quality of PMMW and visual images, compared with some traditional fusion algorithms.

\section{References}

1. Chen, H. M., Lee, S., Rao, R. M., Slamani, M. A., \& Varshney, P. K. (2005). Imaging for concealed weapon detection: a tutorial overview of development in imaging sensors and processing. Signal Processing Magazine, IEEE, 22(2), 52-61.

2. Yujiri, L., Shoucri, M., \& Moffa, P. (2003). Passive millimeter wave imaging.Microwave Magazine, IEEE, 4(3), 39-50.

3. Blum, R. S., \& Liu, Z. (Eds.). (2005). Multi-sensor image fusion and its applications. CRC press. 
4. Smith, M. I., \& Heather, J. P. (2005, March). A review of image fusion technology in 2005. In Defense and Security (pp. 29-45). International Society for Optics and Photonics.

5. Zhang, Z., \& Blum, R. (1997, March). Region-based image fusion scheme for concealed weapon detection. In Proceedings of the 31st Annual Conference on Information Sciences and Systems (pp. 168-173).

6. Lewis, J. J., O'callaghan, R. J., Nikolov, S. G., Bull, D. R., \& Canagarajah, C. N. (2004, June). Region-based image fusion using complex wavelets. InSeventh International Conference on Information Fusion (FUSION) (Vol. 1, pp. 555-562).

7. Liu, Z., Xue, Z., Blum, R. S., \& Laganiere, R. (2006). Concealed weapon detection and visualization in a synthesized image. Pattern analysis and applications, 8(4), 375-389.

8. Xin, W., \& Gaolue, L. (2011, August). Fusion algorithm for infrared-visual image sequences. In Image and Graphics (ICIG), 2011 Sixth International Conference on (pp. 244-248). IEEE.

9. Comaniciu, D., \& Meer, P. (2002). Mean shift: A robust approach toward feature space analysis. Pattern Analysis and Machine Intelligence, IEEE Transactions on, 24(5), 603-619.

10. Da Cunha, A. L., Zhou, J., \& Do, M. N. (2006). The nonsubsampled contourlet transform: theory, design, and applications. Image Processing, IEEE Transactions on, 15(10), 30893101.

11. Rankov, V., Locke, R. J., Edens, R. J., Barber, P. R., \& Vojnovic, B. (2005, March). An algorithm for image stitching and blending. In Proceedings of SPIE (Vol. 5701, pp. 190-199).

12. Manu, V. T., \& Simon, P. (2012). A Novel Statistical Fusion Rule for Image Fusion in Non Subsampled Contourlet Transform Domain. In Advances in Computer Science and Information Technology. Computer Science and Information Technology(pp. 367-376). Springer Berlin Heidelberg.

13. Howell, C., Moore, R., Burks, S., \& Halford, C. (2007, April). An evaluation of fusion algorithms using image fusion metrics and human identification performance. In Defense and Security Symposium (pp. 65430V-65430V). International Society for Optics and Photonics.

14. Xydeas, C. S., \& Petrovic, V. (2000). Objective image fusion performance measure. Electronics Letters, 36(4), 308-309.

15. Li, S., \& Yang, B. (2008). Multifocus image fusion using region segmentation and spatial frequency. Image and Vision Computing, 26(7), 971-979.

16. Qingqing, H., Yuan, J., \& Jian, Y. (2011, June). Improved fusion method for infrared and visible remote sensing imagery using NSCT. In Industrial Electronics and Applications (ICIEA), 2011 6th IEEE Conference on (pp. 1012-1015). IEEE. 